\title{
Triple versus double antithrombotic therapy in patients with atrial fibrillation and stent implantation: a meta-analysis of randomized trials
}

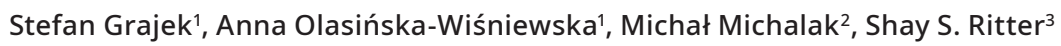 \\ 1 1st Department of Cardiology, Poznan University of Medical Sciences, Poznań, Poland \\ 2 Department of Computer Science and Statistics, Poznan University of Medical Sciences, Poznań, Poland \\ 3 Tel Aviv Sourasky Medical Center, Tel Aviv, Israel
}

\section{KEY WORDS}

atrial fibrillation, dual therapy, stent thrombosis, triple therapy

\section{EDITORIAL}

page 817

\section{Correspondence to:} Anna Olasińska-Wiśniewska, MD, PhD, 1st Department of Cardiology, Poznan University of Medical Sciences, ul. Długa 1/2, 61-848 Poznań, Poland, phone: +48618549146, email: annaolasinska@ump.edu.pl Received: May 19, 2019. Revision accepted: July 12, 2019. Published online: July 16, 2019. Kardiol Pol. 2019; 77 (9): 837-845 doi:10.33963/KP.14899 Copyright by the Author(s), 2019

\begin{abstract}
BACKGROUND Appropriate double (DT) and triple (TT) antithrombotic therapy in patients with atrial fibrillation and stent implantation is unclear.

AIMS The aim of the study was to perform a meta-analysis of studies comparing DT and TT in patients with atrial fibrillation and stent implantation.

METHODS Of the 450 reports, 5 randomized trials were included in the meta-analysis: WOEST, ISAR-REACT, PIONEER AF-PCI, RE-DUAL PCI, and AUGUSTUS, with a total of 9931 patients.

RESULTS Treatment efficacy, as assessed by the incidence of major adverse cardiac events, did not differ significantly between both therapeutic strategies: $8.98 \%$ for DT vs $8.71 \%$ for TT (odds ratio [OR], 1.02; $95 \%$ CI, 0.86-1.21). The incidence of hemorrhagic complications was significantly lower in patients treated with DT than TT (13.1\% and 21.0\%, respectively; OR, 0.57; 95\% CI, 0.47-0.70). In over 90\% of patients, DT included clopidogrel along with an oral anticoagulant (non-vitamin $\mathrm{K}$ antagonist oral anticoagulant or vitamin K antagonist).
\end{abstract}

CONCLUSIONS The results of our meta-analysis are clearly in line with the current trend of the fastest possible reduction in the use of TT in favor of DT. Almost half lower risk of hemorrhagic complications during DT compared with TT, with similar efficacy of the 2 strategies, provides an argument for the wider use of DT in patients with AF and stent implantation.

INTRODUCTION It is well known that in patients with acute coronary syndrome (ACS) undergoing percutaneous coronary intervention (PCI), dual antiplatelet therapy (DAPT) is used for 12 months, with the possibility of shortening it in case of an increased risk of bleeding complications. A similar consensus for patients with stable coronary artery disease (CAD) is 6 months. ${ }^{1}$ In patients with atrial fibrillation (AF), it is necessary to combine oral anticoagulant therapy (OAC), based on vitamin $\mathrm{K}$ antagonists (VKAs) or non-vitamin $\mathrm{K}$ antagonist oral anticoagulants (NOACs), with DAPT as part of triple therapy (TT). ${ }^{2}$ Triple therapy $(\mathrm{VKA}+\mathrm{DAPT})$ provides better protection against thromboembolic complications compared with the single use of a VKA, although the risk of bleeding complications increases by approximately $120 \%$. In turn, double antithrombotic therapy (DT) consisting of a VKA with a single antiplatelet therapy (SAPT) offers worse protection from thromboembolic complications, although the increased risk of hemorrhagic complications is almost halved (about $60 \%){ }^{3}$ Antiplatelet and antithrombotic therapy constitutes even a larger challenge in patients with cancer; however, treatment of this group was described in another study. ${ }^{4}$

The increasing use of radial artery access for angioplasty has significantly reduced the number 


\section{WHAT'S NEW?}

Almost half lower risk of hemorrhagic complications during double compared with triple antithrombotic therapy provides an argument for the wider use of double therapy in patients with atrial fibrillation and stent implantation.

of hemorrhagic complications. On the other hand, second-generation drug-eluting stents (DESs; everolimus, zotarolimus) allowed a radical shortening of DAPT to 3 or even 1 month in patients with sinus rhythm and high risk of hemorrhagic complications. ${ }^{5-8}$ The introduction of stents with an ultrathin strut $(60 \mu)$ enabled a further reduction in the risk of stent thrombosis to $0.9 \% /$ year. $^{9,10}$ Of note, this value is lower than the percentage of bleeding complications during 1 year in patients treated with DAPT. Moreover, it should be emphasized that the improvement in stent implantation technique (high pressures, intravascular ultrasound control, optical coherence tomography) has further reduced the risk of stent thrombosis. These achievements resulted in a more flexible approach to antithrombotic therapy in patients with AF and stent implantation. In practice, this means the possibility of shortening the time of (to as short as possible) and individualizing TT. This fundamental change in the treatment strategy is suggested by the results of trials comparing TT vs DT in patients with AF and stent implantation: WOEST (What is the Optimal Antiplatelet \& Anticoagulant Therapy in Patients With Oral Anticoagulation and Coronary Stenting), ${ }^{11}$ ISAR-TRIPLE (Triple Therapy in Patients on Oral Anticoagulation After Drug Eluting Stent Implantation), ${ }^{12}$ PIONEER AF-PCI (A Study Exploring Two Strategies of Rivaroxaban [JNJ39 039 039; BAY-59-7939] and One of Oral Vitamin K Antagonist in Patients With Atrial Fibrillation Who Undergo Percutaneous Coronary Intervention) ${ }^{13}$ and RE-DUAL PCI (Randomized Evaluation of Dual Antithrombotic Therapy With Dabigatran vs. Triple Therapy With Warfarin in Patients With Nonvalvular Atrial Fibrillation Undergoing Percutaneous Coronary Intervention). ${ }^{14}$ Finally, in March 2019, the results of the AUGUSTUS trial (A Study of Apixaban in Patients With Atrial Fibrillation, Not Caused by a Heart Valve Problem, Who Are at Risk for Thrombosis [Blood Clots] Due to Having Had a Recent Coronary Event, Such as a Heart Attack or a Procedure to Open the Vessels of the Heart ${ }^{15}$ were published, which seems to have finally settled the problem.

The aim of the current study was to present the results of a meta-analysis of randomized trials comparing TT vs DT in patients with AF and stent implantation.

METHODS Search strategy and selection criteria We followed the Preferred Reporting Items for Systematic Reviews and Meta-Analysis
(PRISMA) guidelines for the systematic review and meta-analysis.

The primary exclusion criteria were observational nonrandomized studies, registry data, ongoing trials without results, and duplicate studies. A search was conducted in PubMed, Embase, EBSCO, Cochrane Database of Systematic Reviews, and Web of Science from its inception up to March 2019, using the following search terms in various combinations: "percutaneous coronary intervention", "coronary stenting”, "PCI”, "triple antiplatelet therapy", "dual antiplatelet therapy", "triple therapy”, "dual therapy”, "double therapy”, “vitamin K antagonists", “warfarin”, “dabigatran”, "apixaban”, "rivaroxaban”, “edoxaban”, “aspirin", "thienopyridine", "clopidogrel”, and "randomized clinical trial".

In addition, references of prior systematic reviews and meta-analysis, as well as abstracts from major cardiology meetings, were screened for related studies. Two investigators (SG and MM) independently reviewed the titles, abstracts, and studies to determine their eligibility to meet the inclusion criteria. The same authors independently extracted all the relevant outcomes of interest into a structured data set.

In all studies included in the analysis, hemorrhagic complications were the primary endpoint. Due to the various definitions adopted in the studies, the following indicators of bleeding complications were included in the current meta-analysis: Thrombolysis in Myocardial Infarction (TIMI) major + TIMI minor or International Society on Thrombosis and Haemostasis (ISTH major or clinically relevant nonmajor bleeding) or clinically significant bleeding. The definitions relevant to a particular study are summarized in Supplementary material, Table S1. In each of the studies included in the analysis, the secondary endpoint, defined as a major adverse cardiac event (MACE), was also assessed slightly differently. The relevant definitions for each study are shown in Supplementary material, Table S2. The metadata used for the primary and secondary endpoints in each of the analyzed studies are presented in Supplementary material, Tables $S 3$ and $S 4$.

Statistical analysis The meta-analyses were performed with the use of the DerSimonian and Laird method for random effects. Significant heterogeneity was identified in both analyses, as indicated by the $I^{2}$ values. In general, the $I^{2}$ values of $25 \%, 50 \%$, and $75 \%$ are considered to indicate low, moderate, and high degrees of heterogeneity, respectively. Relative weights are based on the inverse of the observed variance of the treatment effect within studies and between studies. Calculations were performed using Review Manager (RevMan 5.3 Cochrane 


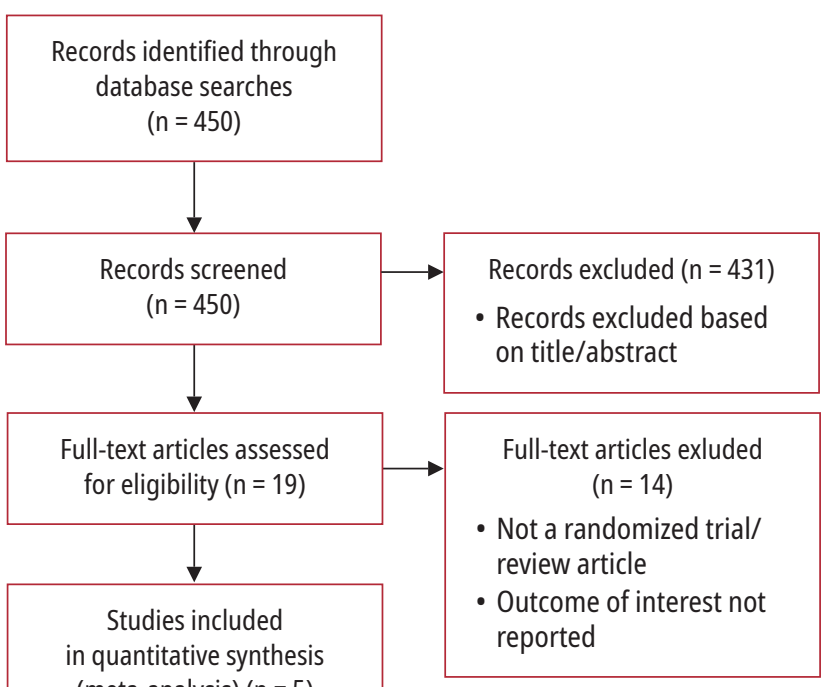

FIGURE 1 Flowchart of literature search
The results showed no presence of publication bias either for safety or efficacy endpoints analyzed in the study (Supplementary material, Figures S1-S8).

RESULTS A total of 450 studies were screened for eligibility, out of which 5 trials with a total of 9931 patients met the inclusion criteria (FIGURE 1) and were included in the final analysis. One arm of the PIONEER AF-PCI trial, ${ }^{13}$ comprising patients treated with low doses of rivaroxaban $(2.5 \mathrm{mg}$, group $2, \mathrm{n}=709$ ), was excluded from the analysis. The most relevant data concerning the characteristics of patients are presented in TABLE 1. The follow-up in the respective studies ranged from 6 to 14 months. Patients with ACS accounted for $30 \%$ to $70 \%$ of the group. The $\mathrm{CHA}_{2} \mathrm{DS}_{2} \mathrm{VASc}$ score ranged from 2.4 to 4.1 , and HAS-BLED, from 2.7 to 3.0 (TABLE1). Diabetes mellitus was present in $23 \%$ to $38 \%$ of patients.

Evaluation of efficacy In both therapeutic strategies, the rate of MACEs was almost identical: $8.98 \%$ and $8.71 \%$ in the DT and TT arms, respectively (odds ratio [OR], 1.02; 95\% CI, 0.86-1.21; FIGURE 2A). In the sensitivity analysis, after exclusion of the ISAR-TRIPLE study ${ }^{12}$ (the DT regimen included acetylsalicylic acid [ASA], whereas in other studies, clopidogrel was used), the respective values were $9.29 \%$ in the DT arm and $8.98 \%$ in the TT arm (OR, 1.02; 95\% CI, 0.851.23; FIGURE 2B). In further data analysis, the studies were divided into 2 subgroups comprising either

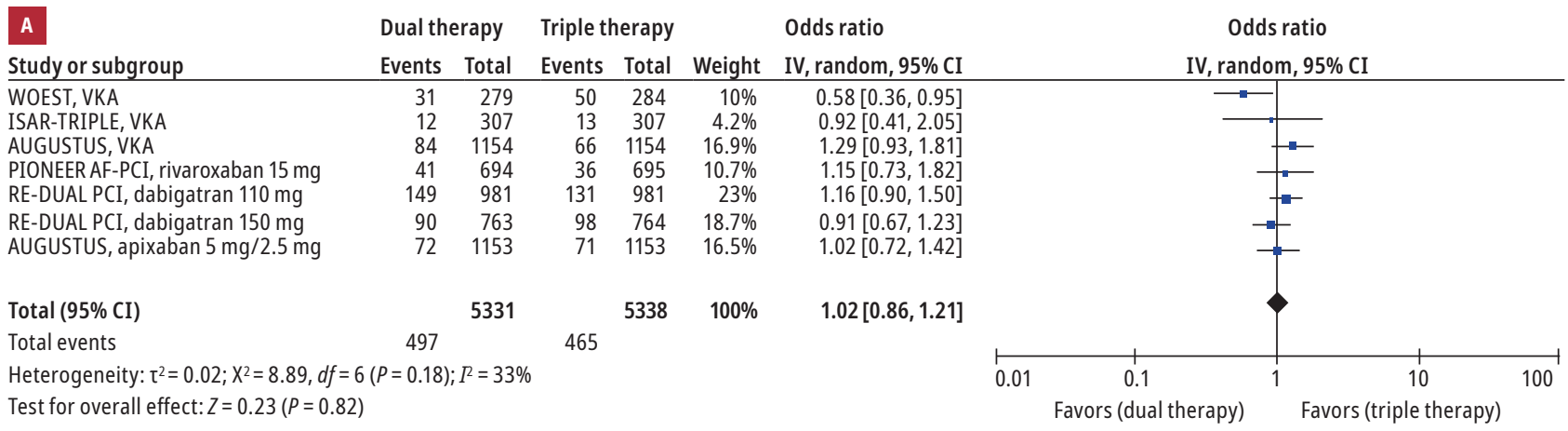

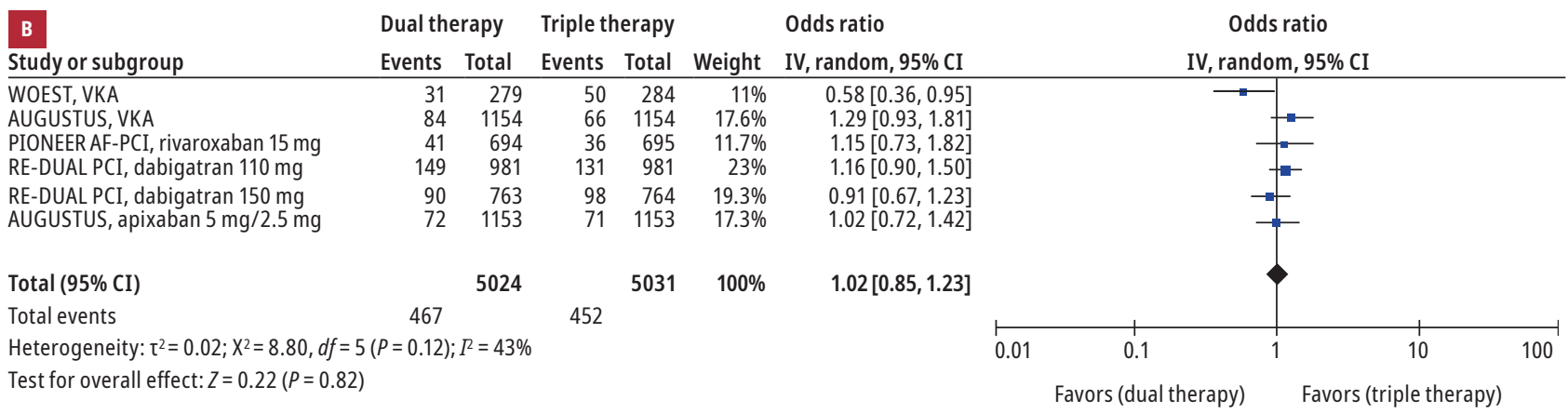


TABLE 1 Baseline characteristics of patients in trials included in the analysis (continued on the next page)

\begin{tabular}{|c|c|c|c|c|c|c|c|}
\hline \multirow{2}{*}{\multicolumn{2}{|c|}{ Characteristics }} & \multicolumn{2}{|c|}{$\begin{array}{r}\text { WOEST, } \\
2013\end{array}$} & \multicolumn{2}{|c|}{$\begin{array}{c}\text { ISAR-TRIPLE, } \\
2015\end{array}$} & \multicolumn{2}{|c|}{$\begin{array}{c}\text { PIONEER-AF PCI, } \\
2016\end{array}$} \\
\hline & & $\begin{array}{l}\text { DT }(n=279) \\
\text { Clopidogrel } \\
\text { (75 mg) } \\
+ \text { VKA }\end{array}$ & $\begin{array}{l}\text { TT }(n=284) \\
\text { Clopidogrel } \\
(75 \mathrm{mg})+\text { ASA } \\
(80-100) \mathrm{mg}+ \\
\text { VKA }\end{array}$ & $\begin{array}{l}\text { 6-week group } \\
(\mathrm{n}=307) \\
\text { ASA }(75-200 \mathrm{mg}) \\
+ \text { VKA } \\
+ \text { clopidogrel } \\
(75 \mathrm{mg}) \text { for } \\
6 \text { weeks }\end{array}$ & $\begin{array}{l}\text { 6-month group } \\
(\mathrm{n}=307) \\
\text { ASA (75-200 mg) } \\
\text { + VKA } \\
\text { + clopidogrel } \\
\text { (75 mg) } \\
\text { for } 6 \text { months }\end{array}$ & $\begin{array}{l}\text { DT }(n=709) \\
\text { Rivaroxaban } \\
(15 \mathrm{mg})+\mathrm{P} \mathrm{Y}_{12} \\
\text { inhibitor } \\
\text { (clopidogrel, } \\
75 \mathrm{mg}, \text { or } \\
\text { ticagrelor, } \\
2 \times 90 \mathrm{mg} \text {, or } \\
\text { prasugrel, } \\
10 \mathrm{mg} \text { ) }\end{array}$ & $\begin{array}{l}\text { TT }(n=706) \\
\text { VKA + ASA } \\
(75-100 \mathrm{mg})+ \\
\text { P2Y inhibitor } \\
\text { (clopidogrel, } \\
75 \mathrm{mg}, \text { or } \\
\text { ticagrelor, } \\
2 \times 90 \mathrm{mg} \text {, or } \\
\text { prasugrel, } \\
10 \mathrm{mg} \text { ) }\end{array}$ \\
\hline \multicolumn{2}{|l|}{ Age, y, mean (SD) } & $70.3(7.0)$ & $69.5(8.0)$ & $73.9(7.7)$ & $73.3(8.7)$ & $70.4(9.1)$ & $69.9(8.7)$ \\
\hline \multicolumn{2}{|l|}{ Female sex, n (\%) } & $65(23)$ & $50(18)$ & $78(25.4)$ & $65(21.2)$ & $181(25.5)$ & $188(26.6)$ \\
\hline \multicolumn{2}{|c|}{$\begin{array}{l}\text { Renal function, } \mathrm{n}(\%) \\
\text { or as indicated otherwise }\end{array}$} & $\begin{array}{l}\text { Renal failure } \\
51 \text { (18) }\end{array}$ & $\begin{array}{l}\text { Renal failure } \\
48 \text { (17) }\end{array}$ & $\begin{array}{l}\text { GFR }<67.3 \mathrm{ml} / \mathrm{min} \\
165(53.7)\end{array}$ & $\begin{array}{l}\text { GFR }<67.3 \mathrm{ml} / \mathrm{min} \\
142(46.3)\end{array}$ & $\begin{array}{l}\text { Mean (SD) } \mathrm{CrCl} \\
78.3(31.3) \mathrm{ml} / \mathrm{min}\end{array}$ & $\begin{array}{l}\text { Mean (SD) } \mathrm{CrCl} \\
80.7(30) \mathrm{ml} / \mathrm{min}\end{array}$ \\
\hline \multicolumn{2}{|c|}{ Type of index event, n (\%) } & ACS, $69(25)$ & ACS, $86(30)$ & $\begin{array}{l}\text { NSTEMI, } 50 \text { (16.3) } \\
\text { STEMI, } 3(1.0) \\
\text { UA, } 49(16) \\
\text { Stable AP, } 205 \text { (66.8) }\end{array}$ & $\begin{array}{l}\text { NSTEMI, } 41 \text { (13.4) } \\
\text { STEMI, } 2 \text { (0.7) } \\
\text { UA, } 52 \text { (16.9) } \\
\text { Stable AP, } 212 \text { (69.1) }\end{array}$ & $\begin{array}{l}\text { NSTEMI, } 130 \text { (18.5) } \\
\text { STEMI, } 86(12.3) \\
\text { UA, } 145(20.7)\end{array}$ & $\begin{array}{l}\text { NSTEMI, } 123 \\
\quad(17.8) \\
\text { STEMI, } 74(10.7) \\
\text { UA, } 164(23.7)\end{array}$ \\
\hline \multicolumn{2}{|c|}{$\mathrm{CHA}_{2} \mathrm{DS}_{2} \mathrm{VASC}$, mean (SD) } & $2.8(1.1)$ & $2.7(1.2)$ & $2.7(1.2)$ & $2.4(1.1)$ & $3.7(1.7)$ & $3.8(1.6)$ \\
\hline \multicolumn{2}{|c|}{$\begin{array}{l}\text { HAS-BLED, mean (SD) } \\
\text { or }<3 \text { vs } \geq 3\end{array}$} & NA & NA & NA & NA & $\begin{array}{l}<3,28 \\
\geq 3,72\end{array}$ & $\begin{array}{l}<3,29 \\
\geq 3,71\end{array}$ \\
\hline \multicolumn{2}{|l|}{ Hypertension, n (\%) } & $193(69)$ & $193(68)$ & $236(76.9)$ & $232(75.6)$ & NA & NA \\
\hline \multicolumn{2}{|c|}{ Diabetes mellitus, n (\%) } & $68(24)$ & $72(25)$ & $85(27.7)$ & $72(23.5)$ & NA & NA \\
\hline \multicolumn{2}{|l|}{ Stroke or TIA, n (\%) } & $49(18)$ & $50(18)$ & $42(13.7)$ & $32(10.4)$ & NA & NA \\
\hline \multicolumn{2}{|c|}{$\begin{array}{l}\text { History of myocardial } \\
\text { infarction, } \mathrm{n}(\%) \text { or as } \\
\text { indicated otherwise }\end{array}$} & $96(34)$ & $100(35)$ & $90(29.3)$ & $76(24.8)$ & $20 \%$ & $22 \%$ \\
\hline \multicolumn{2}{|c|}{ History of CABG, n (\%) } & $56(20)$ & $74(26)$ & $73(23.8)$ & $51(16.6)$ & NA & NA \\
\hline \multicolumn{2}{|l|}{ History of PCI, n (\%) } & $86(31)$ & $101(36)$ & NA & NA & NA & NA \\
\hline \multirow{3}{*}{$\begin{array}{l}\text { Stent type, } \mathrm{n}(\%) \text { or } \\
\text { as indicated } \\
\text { otherwise }\end{array}$} & DES & $181(65)$ & $183(64)$ & $99.4 \%$ & $99 \%$ & $464(65.4)$ & $468(66.5)$ \\
\hline & BMS & $89(32)$ & $86(30)$ & $1(0.3)$ & 0 & $231(32.6)$ & $224(31.8)$ \\
\hline & $\mathrm{DES}+\mathrm{BMS}$ & $3(1)$ & $11(4)$ & $1(0.3)$ & 4 treated lesions ( 1 ) & $14(2.0)$ & $12(1.7)$ \\
\hline
\end{tabular}

Abbreviations: ACS, acute coronary syndrome; ASA, acetylsalicylic acid; BMS, bare metal stent; $C A B G$, coronary artery bypass grafting; creat, creatinine; $C r C l$, creatinine clearance; DES, drug-eluting stent; GFR, glomerular filtration rate; NA, nonapplicable; NSTEMI, non-ST-segment elevation myocardial infarction; PCI, percutaneous intervention; STEMI, ST-segment elevation myocardial infarction; TIA, transient ischemic attack; UA, unstable angina

a VKA or NOAC in both arms (DT and TT). In patients treated with VKA + SAPT (DT) and VKA + DAPT (TT), the MACE rates were $7.29 \%$ and 7.39\%, respectively (OR, 0.9; 95\% CI, 0.56-1.57; FIGURE 3A). The corresponding values in the NOAC-treatment group were $9.8 \%$ and $9.35 \%$ for DT and TT, respectively (OR, 1.059; 95\% CI, 0.901.24; FIGURE 3B).

Safety assessment The incidence of hemorrhagic complications was significantly lower in patients treated with DT than TT: $13.1 \%$ and $21.0 \%$, respectively (OR, 0.57; 95\% CI, 0.47-0.70; FIGURE 4A). After excluding the ISAR-TRIPLE study, ${ }^{12}$ the corresponding values were $13.23 \%$ and $21.7 \%$ (OR, 0.54; 95\% CI, 0.46-0.63;
FIGURE 4B). As in the previous assessment, the studies were divided into 2 subgroups comprising either a VKA or NOAC in both arms (DT and TT). In patients treated with VKA + SAPT (DT) and $\mathrm{VKA}+\mathrm{DAPT}(\mathrm{TT})$, the rates of bleeding complications were $11.5 \%$ and $19.2 \%$, respectively (OR, 0.59; 95\% CI, 0.34-1.04; FIGURE 5A). The corresponding values in patients treated with a NOAC were $13.9 \%$ and $21.9 \%$ (OR, 0.57; 95\% CI, 0.470.69; FIGURE 5B).

DISCUSSION The results of our meta-analysis clearly indicate that, with a similar reduction of MACEs, the risk of bleeding complications in patients treated with DT is $43 \%$ lower than in 
TABLE 1 Baseline characteristics of patients in trials included in the analysis (continued from the previous page)

\begin{tabular}{|c|c|c|c|c|c|c|c|c|}
\hline \multirow{2}{*}{\multicolumn{2}{|c|}{ Characteristics }} & \multicolumn{3}{|c|}{$\begin{array}{c}\text { RE-DUAL PCI, } \\
2017\end{array}$} & \multicolumn{4}{|c|}{$\begin{array}{c}\text { AUGUSTUS, } \\
2019\end{array}$} \\
\hline & & $\begin{array}{l}\text { DT }(\mathrm{n}=981) \\
\text { Dabigatran } \\
(2 \times 110 \mathrm{mg}) \\
+\mathrm{P} 2 \mathrm{Y}_{12} \\
\text { inhibitor } \\
\text { (clopidogrel, } \\
75 \mathrm{mg}, \text { or } \\
\text { ticagrelor, } \\
2 \times 90 \mathrm{mg})\end{array}$ & $\begin{array}{l}\text { DT }(n=763) \\
\text { Dabigatran } \\
(2 \times 150 \mathrm{mg}) \\
+\mathrm{P}^{2} \mathrm{Y}_{12} \\
\text { inhibitor } \\
\text { (clopidogrel, } \\
75 \mathrm{mg}, \text { or } \\
\text { ticagrelor, } \\
2 \times 90 \mathrm{mg})\end{array}$ & $\begin{array}{l}\text { TT }(\mathrm{n}=981) \\
\text { VKA + ASA } \\
(<100 \mathrm{mg}) \\
+\mathrm{P}^{\mathrm{Y}} \mathrm{Y}_{12} \\
\text { inhibitor } \\
\text { (clopidogrel, } \\
75 \mathrm{mg}, \text { or } \\
\text { ticagrelor, } \\
2 \times 90 \mathrm{mg})\end{array}$ & $\begin{array}{l}\text { DT/TT }(n=2306) \\
\text { Apixaban } \\
(2 \times 5 \mathrm{mg} \\
\text { or } 2 \times 2.5 \mathrm{mg}) \\
+\mathrm{P}^{2} \mathrm{Y}_{12} \text { inhibitor } \\
+ \text { ASA, } 81 \mathrm{mg} / \\
\text { placebo }\end{array}$ & $\begin{array}{l}\text { DT/TT } \\
(n=2308) \\
\text { VKA + P2Y } \\
\text { inhibitor + ASA, } \\
\quad 81 \mathrm{mg} / \text { placebo }\end{array}$ & $\begin{array}{l}\text { DT/TT } \\
(\mathrm{n}=2307) \\
\text { Apixaban } \\
\quad(2 \times 5 \mathrm{mg} \text { or } \\
2 \times 2.5 \mathrm{mg}) \\
\text { /VKA } \\
+ \text { P2Y } \\
\text { inhibitor } \\
+ \text { ASA, } \\
81 \mathrm{mg}\end{array}$ & $\begin{array}{l}\text { DT/TT } \\
(\mathrm{n}=2307) \\
\text { Apixaban } \\
(2 \times 5 \mathrm{mg} \text { or } \\
2 \times 2.5 \mathrm{mg}) / \\
\text { VKA + P2Y } \\
\text { inhibitor } \\
\text { + placebo }\end{array}$ \\
\hline \multicolumn{2}{|c|}{ Age, y, mean (SD) } & $71.5(8.9)$ & $68.6(7.7)$ & $71.7(8.9)$ & 70.4 & 70.9 & 70.8 & 70.6 \\
\hline \multicolumn{2}{|c|}{ Female sex, n (\%) } & $253(25.8)$ & $171(22.4)$ & $231(23.5)$ & $670(29.1)$ & $667(28.9)$ & $696(30.2)$ & $641(27.8)$ \\
\hline \multicolumn{2}{|c|}{$\begin{array}{l}\text { Renal function, } \mathrm{n}(\%) \\
\text { or as indicated otherwise }\end{array}$} & $\begin{array}{l}\text { Mean (SD) } \mathrm{CrCl} \text {, } \\
76.3 \\
(28.9) \mathrm{ml} / \mathrm{min}\end{array}$ & $\begin{array}{l}\text { Mean (SD) } \\
\quad \mathrm{CrCl}, 83.7 \\
(31) \mathrm{ml} / \mathrm{min}\end{array}$ & $\begin{array}{l}\text { Mean (SD) CrCl, } \\
75.4(29.1) \\
\mathrm{ml} / \mathrm{min}\end{array}$ & $\begin{array}{l}\text { creat } \geq 1.5 \mathrm{mg} / \mathrm{dl}, \\
173(7.6)\end{array}$ & $\begin{array}{l}\text { creat } \geq 1.5 \mathrm{mg} / \mathrm{dl}, \\
207(9.2)\end{array}$ & $\begin{array}{l}\text { creat } \geq 1.5 \\
\mathrm{mg} / \mathrm{dl}, 182 \\
(8.1)\end{array}$ & $\begin{array}{l}\text { creat } \\
\geq 1.5 \mathrm{mg} / \mathrm{dl} \\
198(8.7)\end{array}$ \\
\hline \multirow{5}{*}{\multicolumn{2}{|c|}{ Type of index event, n (\%) }} & ACS, $509(51.9)$ & ACS, 391 (51.2) & ACS, 475 (48.4) & \multirow{3}{*}{$\begin{array}{l}\text { ACS and PCI, } 873 \\
(38) \\
\text { ACS - medical } \\
\text { therapy, } 547 \\
(23.8)\end{array}$} & \multirow{3}{*}{$\begin{array}{l}\text { ACS and PCI, } \\
841(36.6) \\
\text { ACS - medical } \\
\text { therapy, } 550 \\
(23.9)\end{array}$} & \multirow{4}{*}{$\begin{array}{l}\text { ACS and PCI, } \\
844(36.8) \\
\text { ACS - } \\
\text { medical } \\
\text { therapy, } \\
547(23.9)\end{array}$} & $\begin{array}{c}\text { ACS and PCI, } \\
870(37.8)\end{array}$ \\
\hline & & $\begin{array}{l}\text { Stable angina, } \\
433 \text { (44.1) }\end{array}$ & $\begin{array}{l}\text { Stable angina, } \\
320(41.9)\end{array}$ & $\begin{array}{l}\text { Stable angina, } \\
429(43.7)\end{array}$ & & & & \multirow{2}{*}{$\begin{array}{l}\text { ACS - medical } \\
\text { therapy, } 550 \\
(23.9)\end{array}$} \\
\hline & & $\begin{array}{l}\text { Staged PCI, } 156 \\
(15.9)\end{array}$ & $\begin{array}{r}\text { Staged PCI, } \\
138(18.1)\end{array}$ & $\begin{array}{l}\text { Staged PCI, } \\
168(17.1)\end{array}$ & & & & \\
\hline & & \multirow{2}{*}{ Other, 43 (4.4) } & \multirow{2}{*}{ Other, 65 (8.5) } & \multirow{2}{*}{ Other, 62 (6.3) } & \multirow{2}{*}{$\begin{array}{l}\text { Elective PCI, } 877 \\
\quad(38.2)\end{array}$} & \multirow{2}{*}{$\begin{array}{l}\text { Elective PCI, } 907 \\
\quad(39.5)\end{array}$} & & \multirow{2}{*}{$\begin{array}{l}\text { Elective PCI, } \\
882(38.3)\end{array}$} \\
\hline & & & & & & & $\begin{array}{l}\text { Elective PCI, } \\
902 \text { (39.3) }\end{array}$ & \\
\hline \multicolumn{2}{|c|}{$\mathrm{CHA}_{2} \mathrm{DS}_{2} \mathrm{VASC}$, mean (SD) } & $3.7(1.6)$ & $3.3(1.5)$ & $3.8(1.5)$ & $3.9(1.6)$ & $4.0(1.6)$ & $3.9(1.6)$ & $3.9(1.6)$ \\
\hline \multicolumn{2}{|c|}{$\begin{array}{l}\text { HAS-BLED, mean (SD) } \\
\text { or }<3 \text { vs } \geq 3\end{array}$} & $2.7(0.7)$ & $2.6(0.7)$ & $2.8(0.8)$ & $2.9(1.0)$ & $2.9(0.9)$ & $2.8(0.9)$ & $2.9(1.0)$ \\
\hline \multicolumn{2}{|c|}{ Hypertension, n (\%) } & NA & NA & NA & 2042 (88.6) & $2031(88)$ & $2031(88)$ & $2042(88.5)$ \\
\hline \multicolumn{2}{|c|}{ Diabetes mellitus, n (\%) } & $362(36.9)$ & $260(34.1)$ & $371(37.9)$ & $842(36.5)$ & $836(36.2)$ & $842(36.5)$ & $836(36.2)$ \\
\hline \multicolumn{2}{|c|}{ Stroke or TIA, n (\%) } & $74(7.5)$ & $52(6.8)$ & $100(10.2)$ & $326(14.2)$ & $307(13.4)$ & $297(13.0)$ & $336(14.7)$ \\
\hline \multicolumn{2}{|c|}{$\begin{array}{l}\text { History of myocardial } \\
\text { infarction, n (\%) or as } \\
\text { indicated otherwise }\end{array}$} & $237(24.2)$ & $194(25.4)$ & $268(27.3)$ & NA & NA & NA & NA \\
\hline \multicolumn{2}{|c|}{ History of CABG, $n(\%)$} & $97(9.9)$ & $79(10.4)$ & $111(11.3)$ & NA & NA & NA & NA \\
\hline \multicolumn{2}{|c|}{ History of PCI, n (\%) } & $326(33.2)$ & $239(31.3)$ & $347(35.4)$ & NA & NA & NA & NA \\
\hline \multirow{3}{*}{$\begin{array}{l}\text { Stent type, } \mathrm{n} \\
(\%) \text { or as } \\
\text { indicated } \\
\text { otherwise }\end{array}$} & DES & $804(82.1)$ & $621(81.5)$ & $826(84.6)$ & \multirow[t]{3}{*}{ NA } & \multirow[t]{3}{*}{ NA } & \multirow[t]{3}{*}{ NA } & \multirow[t]{3}{*}{ NA } \\
\hline & BMS & $148(15.1)$ & $123(16.1)$ & $133(13.6)$ & & & & \\
\hline & $\mathrm{DES}+\mathrm{BMS}$ & $19(1.9)$ & $10(1.3)$ & $12(1.2)$ & & & & \\
\hline
\end{tabular}

Abbreviations: ACS, acute coronary syndrome; ASA, acetylsalicylic acid; BMS, bare metal stent; CABG, coronary artery bypass grafting; creat, creatinine; CrCl, creatinine clearance; DES, drug-eluting stent; GFR, glomerular filtration rate; NA, nonapplicable; NSTEMI, non-ST-segment elevation myocardial infarction; PCI, percutaneous intervention; STEMI, ST-segment elevation myocardial infarction; TIA, transient ischemic attack; UA, unstable angina

the TT arm. The $\mathrm{CHA}_{2} \mathrm{DS}_{2}$ VASc scores in the analyzed studies were similar in AUGUSTUS, ${ }^{15} \mathrm{RE}$ -DUAL PCI, ${ }^{14}$ and PIONEER AF-PCI ${ }^{13}$ and ranged from 3.3 to 4.1 . Slightly lower rates (2.4-2.7) were noted in the WOEST ${ }^{11}$ and ISAR-TRIPLE ${ }^{12}$ trials. In studies that presented the HAS-BLED score, the index values ranged from 2.7 to 3 , so the populations were largely similar in terms of the risk of cardiovascular events and bleeding complications. New antiplatelet drugs (P2Y 12 inhibitors), ticagrelor in particular, were used extremely rarely, including $12 \%$ in the RE-DUAL
PCI trial, ${ }^{14} 4 \%$ in the PIONEER AF-PCI trial, ${ }^{13}$ and $5 \%$ in the AUGUSTUS trial. ${ }^{15}$ In the WOEST ${ }^{11}$ and ISAR-TRIPLE ${ }^{12}$ trials, the new $\mathrm{P}_{2} \mathrm{Y}_{12}$ inhibitors were not used. In both the DT and TT arms, over $90 \%$ of patients enrolled in the meta-analysis used clopidogrel. A slightly higher percentage of ticagrelor in the RE-DUAL PCI study does not allow a recommendation of new potent P2 $\mathrm{Y}_{12}$ inhibitors in chronic DT and TT treatment. This view is in line with current guidelines. ${ }^{1}$

Both in patients treated with VKAs and in those treated with NOACs, there were no 


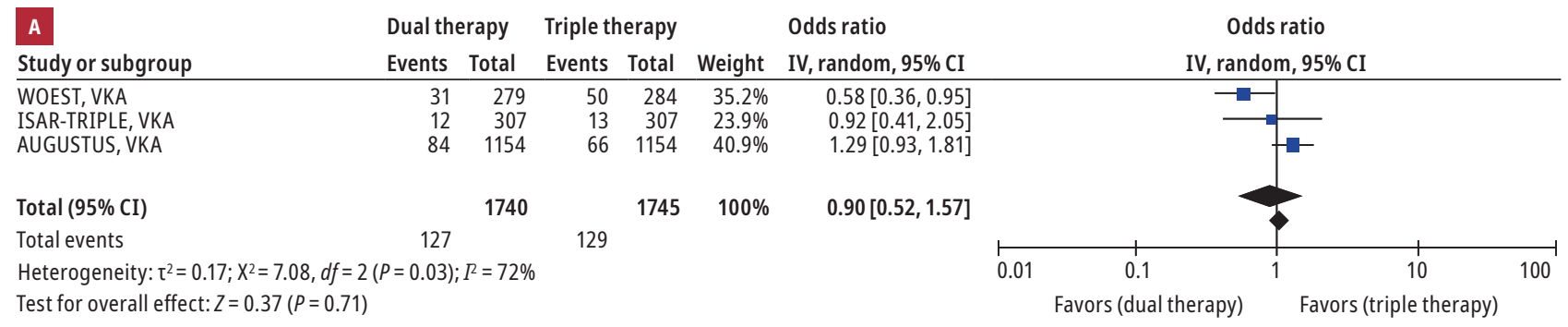

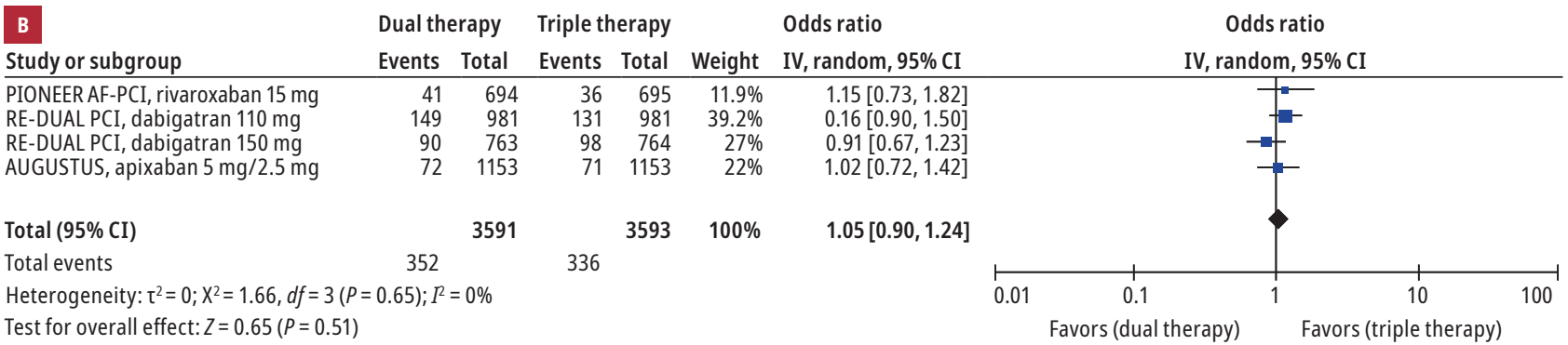

FIGURE 3 Meta-analysis results for secondary efficacy endpoints: A - a substudy for vitamin K antagonist trials; B - a substudy for non-vitamin K antagonist oral anticoagulant trials

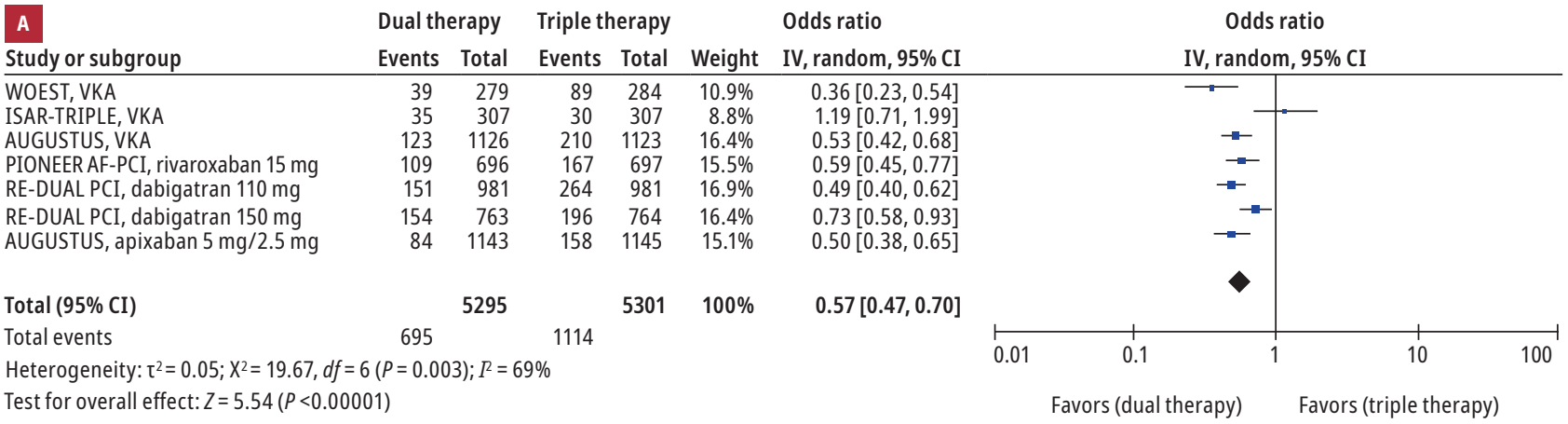

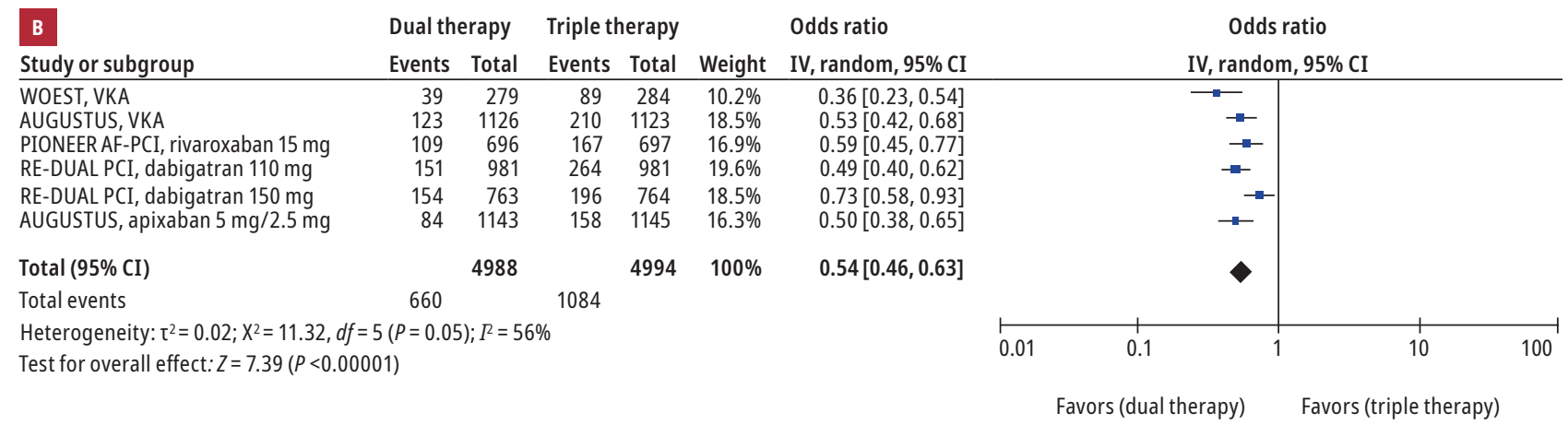

FIGURE 4 Meta-analysis results for primary safety endpoints: A - all trials; $\mathbf{B}$ - without the ISAR-REACT trial

significant differences in the frequency of MACEs between the DT and TT arms (FIGURES 2 and 3), while in both subgroups (VKA and NOAC), we observed a lower risk of bleeding complications in DT vs TT arms: $41 \%$ and $43 \%$, respectively (FIGURES 4 and 5). Therefore, regardless of the basal anticoagulant treatment (VKA or NOAC), the DT compared with TT strategy significantly reduces the risk of bleeding complications, with an unchanged effect on ischemic cardiovascular events.
Treatment with NOACs or VKAs in combination with SAPT may raise some objections to the DAPT strategy commonly recommended after stent implantation, especially in patients with ACS. In the studies included in the meta-analysis, patients with ACS accounted for only $25 \%$ to $30 \%$ of the population in both arms of the WOEST study ${ }^{11}$ and $30 \%$ to $33 \%$ in the ISAR-TRIPLE study. ${ }^{12}$ In the PIONEER AF-PCI ${ }^{13}$ and RE-DUAL ${ }^{14}$ studies, patients with ACS constituted 


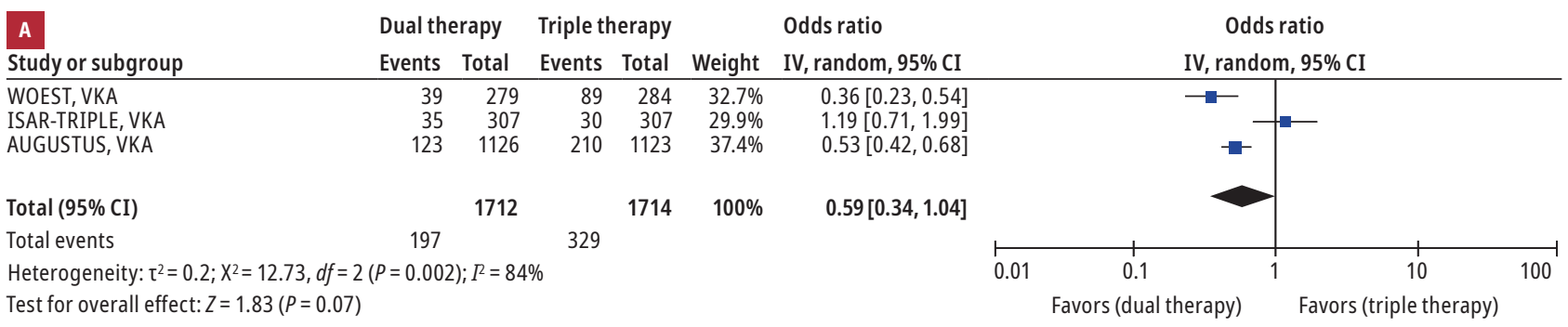

\begin{tabular}{|c|c|c|c|c|c|c|c|c|c|c|c|}
\hline B & Dual th & erapy & Triple tl & nerapy & & Odds ratio & & & $s$ & & \\
\hline Study or subgroup & Events & Total & Events & Total & Weight & IV, random, 95\% CI & & & & & \\
\hline $\begin{array}{l}\text { PIONEER AF-PCI, rivaroxaban } 15 \mathrm{mg} \\
\text { RE-DUAL PCI, dabigatran } 110 \mathrm{mg} \\
\text { RE-DUAL PCI, dabigatran } 150 \mathrm{mg} \\
\text { AUGUSTUS, apixaban } 5 \mathrm{mg} / 2.5 \mathrm{mg}\end{array}$ & $\begin{array}{r}109 \\
151 \\
154 \\
84\end{array}$ & $\begin{array}{r}696 \\
981 \\
763 \\
1143\end{array}$ & $\begin{array}{l}167 \\
264 \\
196 \\
158\end{array}$ & $\begin{array}{r}697 \\
981 \\
764 \\
1145\end{array}$ & $\begin{array}{l}23.6 \% \\
27.6 \% \\
26.1 \% \\
22.8 \%\end{array}$ & $\begin{array}{l}0.59[0.45,0.77] \\
0.49[0.40,0.62] \\
0.73[0.58,0.93] \\
0.50[0.38,0.65]\end{array}$ & & & & & \\
\hline Total $(95 \% \mathrm{CI})$ & & 3583 & & 3587 & $100 \%$ & $0.57[0.47,0.69]$ & & & & & \\
\hline Total events & 498 & & 785 & & & & & & & & \\
\hline Heterogeneity: $\tau^{2}=0.02 ; X^{2}=6.81, a$ & $P=0.08)$ & $I^{2}=56 \%$ & & & & & 0.01 & 0.1 & & 10 & 100 \\
\hline Test for overall effect: $Z=5.8(P<0.0$ & & & & & & & & (dual & & ple t & \\
\hline
\end{tabular}

FIGURE 5 Meta-analysis results for primary safety endpoints: A - a substudy for vitamin K antagonist trials; B - substudy for non-vitamin Kantagonist oral anticoagulant trials

half of the population in both arms (TABLE1), and all of them were treated with PCI. The highest number of patients with ACS was included in the AUGUSTUS study ${ }^{15}$ (about 60\%-61\%), but only $36 \%$ and $38 \%$ of patients in the VKA and NOAC arms, respectively, were treated with PCI, while the remaining patients were treated conservatively. It is well known that in patients with ACS, increased platelet activity is observed especially in the first 30 days after stent implantation, which results in an increased risk of early stent thrombosis with the highest mortality risk. ${ }^{16}$

While intuitively we are inclined to prefer DT in patients with stable CAD starting from the first or second day after PCI, in ACS early DAPT cessation and the use of NOAC or VKA + SAPT may raise concerns. In the PIONEER AF-PCI, ${ }^{13}$ RE-DUAL, ${ }^{14}$ and WOEST ${ }^{11}$ trials, patients received DT 2 to 3 days after PCI (without aspirin, which was only given in the first 24 to 48 hours). In the AUGUSTUS trial, ${ }^{15}$ this period (the median time from the index event to randomization) was 6 days. An important clinical observation is that in these studies, TT was not even used for 1 month after stent implantation. Only in the ISAR-TRIPLE trial, ${ }^{12} \mathrm{TT}$ was administered for 1 month ( 6 weeks) in the experimental arm (DT arm) and then converted to DT until the end of the 9-month follow-up. However, in this study, about two-thirds of patients in both arms (TT and DT) were diagnosed with stable CAD. ${ }^{12}$ None of the mentioned studies included a separate assessment of DT and TT in patients with ACS and stable $\mathrm{CAD}$, which constitutes a limitation to drawing definite conclusions.

Even greater doubts may be raised by the fact that in the RE-DUAL study, a marked, although nonsignificant, increase in the number of patients with myocardial infarction as well as stent thrombosis was noted with a 110-mg dose of dabigatran twice daily with clopidogrel when compared with TT. ${ }^{14}$ The latest European Society of Cardiology (ESC) guidelines on revascularization ${ }^{1}$ recommend a 150-mg dose of dabigatran in patients treated with PCI. In the first PIONEER AF-PCI study, ${ }^{13}$ due to the careful search for an optimal TT strategy in patients with AF and stent implantation, the dose of rivaroxaban was lower $(15 \mathrm{mg})$ than recommended for patients with $\mathrm{AF}(20 \mathrm{mg})$.

Differences in study protocols raise questions to which we have no definite answers. Frequent changes in the ESC and the European Heart Rhythm Association (EHRA) guidelines reflect this uncertainty. In the joint consensus published in 2018, ${ }^{17}$ it is recommended to implement DT directly after PCI 11,13-15 or TT for only 1 month and then chronically DT ${ }^{12}$ in patients with AF after stent implantation and with an increased risk of bleeding complications. In patients with an increased risk of ischemic complications (ACS), the consensus proposes TT for 3 to 6 months and then DT up to the 12th month. Therefore, attention was paid to the possibility of shortening TT to 3 months. However, for patients with ACS and a high risk of bleeding complications, this period can be dangerously long. As stated above, in the PIONEER AF-PCI ${ }^{13}$ and RE-DUAL ${ }^{14}$ trials, ACS patients constituted half of the participants, and DT (NOAC + SAPT) was used after PCI and the completion of intravenous anticoagulant therapy action. Improvement of stent implantation techniques and the use of third-generation DESs (ultrathin struts) ${ }^{9,10}$ have significantly reduced the risk of stent thrombosis. In clinical 
practice, the choice of treatment in patients with $\mathrm{AF}$ and an implanted stent in both stable CAD, and especially in ACS, is much different. ${ }^{18,19}$ Individualization of treatment seems to be the key to rational therapeutic decision making. ${ }^{20}$

It seems that in patients with $\mathrm{AF}$ and stent implantation, DT implemented immediately after PCI (24-48 hours) will replace TT in a subgroup of patients with stable CAD. In patients with ACS with a too high risk of bleeding complications, TT will be used for 1 to 3 months and then converted to DT for chronic treatment.

Hamlet's question remains: which of the antiplatelet drugs (SAPT) will be a component of DT? As previously mentioned, the experience from the RE-DUAL PCI trial, ${ }^{14}$ in which $12 \%$ of patients used ticagrelor in combination with dabigatran, is insufficient to recommend a new strong $\mathrm{P}_{2} \mathrm{Y}_{12}$ in combination with a NOAC or VKA in DT as well as in TT. Thus, ASA or clopidogrel? In the WOEST study, ${ }^{11}$ in patients treated with DT (clopidogrel + VKA) compared with TT (ASA, clopidogrel, and VKA), a greater reduction of the secondary composite ischemic endpoint (hazard ratio [HR], 0.60 [95\% CI, 0.38-0.94]) was observed. Also, the primary endpoint (bleeding complications) was significantly less frequent in patients treated with DT vs TT (HR, 0.36 [95\% CI, 0.26-0.50]). In a Scandinavian registry, ${ }^{21}$ in patients with $\mathrm{AF}$ and myocardial infarction after stent implantation, the greatest benefits (reduction of cardiovascular mortality, total mortality, myocardial infarction, and stroke) with similar indices of bleeding complications were observed in patients treated with VKA + clopidogrel vs VKA, clopidogrel, and ASA. In turn, in the ADAPT-DES study (Assessment of Dual Antiplatelet Therapy With Drug-Eluting Stents ${ }^{22}$ in patients with sinus rhythm, after second-generation DES implantation (zotarolimus), ASA was added to clopidogrel (resistance to both drugs was evaluated). After 12 months, it had no effect on the reduction of ischemic complications (stent thrombosis, myocardial infarction), whereas a significant increase in bleeding complications was observed. Recently, at the American Heart Association Congress in New Orleans, the results of 2 randomized studies were presented (STOPDAPT-2 [Short and Optimal Duration of Dual Antiplatelet Therapy After Everolimus-Eluting Cobalt-Chromium Stent-2] and SMART-CHOICE [Comparison Between P2Y12 Antagonist Monotherapy and Dual Antiplatelet Therapy After DES]) comparing DAPT (ASA + clopidogrel) vs SAPT (clopidogrel after 1 or 3 months of ASA withdrawal). In the STOPDAPT-2 study, after 12 months of follow-up, the net clinical endpoint (ischemic + hemorrhagic complications) was lower in the group treated with clopidogrel vs DAPT (2.4\% and $3.7 \%$ respectively, $P<0.04) .^{23}$ In the SMART-CHOICE study, there were no significant differences in MACEs between the arm treated with clopidogrel monotherapy vs DAPT (2.9\% vs $2.5 \%$ ). Hemorrhagic complications (Bleeding Academic Research Consortium [BARC] 2-5 bleeding) were observed less frequently with clopidogrel therapy than with DAPT ( $2.0 \%$ and $3.4 \%$, respectively, $P<0.04) .{ }^{24}$ In the CAPRIE study, ${ }^{25}$ in patients at high risk of ischemic complications treated with clopidogrel compared with ASA, a significant reduction in the composite endpoint (cardiovascular death, myocardial infarction, stroke) and significantly less frequent gastrointestinal bleeding complications were observed. Already in 2014, a joint consensus of the EHRA and ESC preferred the use of clopidogrel in DT in patients with AF after stent implantation. ${ }^{26}$ When analyzing the results of our meta-analysis, it may be noted that only in the ISAR-TRIPLE trial, ${ }^{12}$ there was no significant reduction in hemorrhagic complications in the DT group compared with TT (FIGURES $4 \mathrm{~A}$ and $5 \mathrm{~A}$ ). In this study, the experimental arm (DT) was based on the combination of VKA with ASA vs VKA + ASA + clopidogrel.

Conclusions In patients with $\mathrm{AF}$ and stent implantation, the results of our meta-analysis are clearly in line with the current trend of the fastest possible reduction in the use of TT in favor of DT. Due to the increased risk of bleeding complications (the need for anticoagulation), the duration of DT should be considered individually depending on the risk assessment of ischemic complications (ACS, Global Registry of Acute Coronary Events [GRACE] score >140, and SYNTAX score), and bleeding complications.

\section{SUPPLEMENTARY MATERIAL}

Supplementary material is available at www.mp.pl/kardiologiapolska.

\section{ARTICLE INFORMATION}

\section{CONFLICT OF INTEREST None declared.}

OPEN ACCESS This is an Open Access article distributed under the terms of the Creative Commons Attribution-NonCommercial-NoDerivatives 4.0 International License (CC BY-NC-ND 4.0), allowing third parties to download articles and share them with others, provided the original work is properly cited, not changed in any way, distributed under the same license, and used for noncommercial purposes only. For commercial use, please contact the journal office at kardiologiapolska@ptkardio.pl.

HOW TO CITE Grajek S, Olasińska-Wiśniewska A, Michalak M, Ritter SS. Triple versus double antithrombotic therapy in patients with atrial fibrillation and stent implantation: a meta-analysis of randomized trials. Kardiol Pol. 2019; 77: 837-845. doi:10.33963/KP.14899

\section{REFERENCES}

1 Neumann F], Sousa-Uva M, Ahlsson A, et al; ESC Scientific Document Group. 2018 ESC/EACTS Guidelines on myocardial revascularization. Eur Heart J. 2019; 40: 87-165.

2 Crowther MA, Eikelboom JW. Dual and triple antithrombotic therapies: current patterns of practice and controversies. Kardiol Pol. 2018; 76: 937-944.

3 Heidbuchel H, Verhamme P, Alings M, et al. Updated European Heart Rhythm Association Practical Guide on the use of non-vitamin K antagonist anticoagulants in patients with non-valvular atrial fibrillation. Europace. 2015; 17: 1467-1507.

4 Banasiak W, Zymliński R, Undas A. Optimal management of cancer patients with acute coronary syndrome. Pol Arch Intern Med. 2018; 128: 244-253.

5 Palmerini T, Sangiorgi D, Valgimigli M, et al. Short- versus long-term dual antiplatelet therapy after drug-eluting stent implantation: an individual patient data pairwise and network meta-analysis. J Am Coll Cardiol. 2015; 65: 1092-1102. 
6 Palmerini T, Biondi-Zoccai G, Della Riva D, et al. Stent thrombosis with drug-eluting and bare-metal stents: evidence from a comprehensive network meta-analysis. Lancet. 2012; 379: 1393-1402.

7 Sen H, Lam MK, Löwik MM, et al. Clinical events and patient-reported chest pain in all-comers treated with resolute integrity and promus element stents: 2-year follow-up of the DUTCH PEERS (DUrable Polymer-Based STent CHallenge of Promus ElemEnt Versus ReSolute Integrity) randomized trial (TWENTE II). JACC Cardiovasc Interv. 2015; 8: 889-899.

8 Giustino G, Baber U, Sartori S, et al. Duration of dual antiplatelet therapy after drug-eluting stent implantation: a systematic review and meta-analysis of randomized controlled trials. J Am Coll Cardiol. 2015; 65: 1298-1310.

9 Bangalore S, Toklu B, Patel N, et al. Newer-generation ultrathin strut drug-eluting stents versus older second-generation thicker strut drug-eluting stents for coronary artery disease. Circulation. 2018; 138: 2216-2226.

10 Zaman A, de Winter RJ, Kogame N, et al. Safety and efficacy of a sirolimus-eluting coronary stent with ultra-thin strut for treatment of atherosclerotic lesions (TALENT): a prospective multicentre randomised controlled trial. Lancet. 2019; 393: 987-997.

11 Dewilde WJ, Oirbans T, Verheugt FW, et al; WOEST study investigators. Use of clopidogrel with or without aspirin in patients taking oral anticoagulant therapy and undergoing percutaneous coronary intervention: an open-label, randomised, controlled trial. Lancet. 2013; 381: 1107-1115.

12 Fiedler KA, Maeng M, Mehilli J, et al. Duration of triple therapy in patients requiring oral anticoagulation after drug-eluting stent implantation: the ISAR-TRIPLE trial. J Am Coll Cardiol. 2015; 65: 1619-1629.

13 Gibson CM, Mehran R, Bode C, et al. Prevention of bleeding in patients with atrial fibrillation undergoing PCI. N Engl J Med. 2016; 375: 2423-2434.

14 Cannon CP, Bhatt DL, Oldgren J, et al; RE-DUAL PCI Steering Committee and Investigators. Dual antithrombotic therapy with dabigatran after PCI in atrial fibrillation. N Engl J Med. 2017; 377: 1513-1524.

15 Lopes RD, Heizer G, Aronson R, et al; AUGUSTUS Investigators. Antithrombotic therapy after acute coronary syndrome or PCI in atrial fibrillation. $\mathrm{N}$ Engl J Med. 2019; 380: 1509-1524.

16 Secemsky EA, Matteau A, Yeh RW, et al; PROTECT Trial Investigators. Comparison of short- and long-term cardiac mortality in early versus late stent thrombosis (from Pooled PROTECT Trials). Am J Cardiol. 2015; 115: 1678-1684.

17 Lip GYH, Collet JP, Haude M, et al; ESC Scientific Document Group. 2018 Joint European consensus document on the management of antithrombotic therapy in atrial fibrillation patients presenting with acute coronary syndrome and/or undergoing percutaneous cardiovascular interventions: a joint consensus document of the European Heart Rhythm Association (EHRA), European Society of Cardiology Working Group on Thrombosis, European Association of Percutaneous Cardiovascular Interventions (EAPCI), and European Association of Acute Cardiac Care (ACCA) endorsed by the Heart Rhythm Society (HRS), Asia-Pacific Heart Rhythm Society (APHRS), Latin America Heart Rhythm Society (LAHRS), and Cardiac Arrhythmia Society of Southern Africa (CASSA). Europace. 2019; 21: 192-193.

18 Sindet-Pedersen C, Lamberts M, Staerk L, et al. Combining Oral Anticoagulants With Platelet Inhibitors in Patients With Atrial Fibrillation and Coronary Disease. J Am Coll Cardiol. 2018; 72: 1790-1800.

19 Sherwood MW, Cyr DD, Jones WS, et al. Use of Dual Antiplatelet Therapy and Patient Outcomes in Those Undergoing Percutaneous Coronary Intervention: The ROCKET AF Trial. JACC Cardiovasc Interv. 2016; 9: 1694-1702.

20 Montalescot G, Angiolillo DJ. Anticoagulation, the unknown of the antithrombotic equation after stenting of an acute coronary syndrome. J Am Coll Cardiol. 2019; 73: 775-778.

21 Lamberts M, Gislason GH, Olesen JB, et al. Oral anticoagulation and antiplatelets in atrial fibrillation patients after myocardial infarction and coronary intervention. J Am Coll Cardiol. 2013; 62: 981-998.

22 Stone GW, Witzenbichler B, Weisz G, et al; ADAPT-DES Investigators. Platelet reactivity and clinical outcomes after coronary artery implantation of drug-eluting stents (ADAPT-DES): a prospective multicentre registry study. Lancet. 2013; 382: 614-623.

23 Watanabe H, Domei T, Morimoto T, et al; STOPDAPT-2 Investigators. Effect of 1-month dual antiplatelet therapy followed by clopidogrel vs 12-month dual antiplatelet therapy on cardiovascular and bleeding events in patients receiving pci: the STOPDAPT-2 randomized clinical trial. JAMA. 2019; 321: 2414-2427.

24 Hahn JY, Song YB, Oh JH, et al; SMART-CHOICE Investigators. Effect of P2Y12 inhibitor monotherapy vs dual antiplatelet therapy on cardiovascular events in patients undergoing percutaneous coronary intervention: the SMART-CHOICE randomized clinical trial. JAMA. 2019; 321: 2428-2437.

25 CAPRIE Steering Committee. A randomised, blinded, trial of clopidogrel versus aspirin in patients at risk of ischaemic events (CAPRIE). CAPRIE Steering Committee. Lancet. 1996; 348: 1329-1339.

26 Lip GY, Windecker S, Huber K, et al. Management of antithrombotic therapy in atrial fibrillation patients presenting with acute coronary syndrome and/or undergoing percutaneous coronary or valve interventions: a joint consensus document of the European Society of Cardiology Working Group on Thrombosis, European Heart Rhythm Association (EHRA), European Association of Percutaneous Cardiovascular Interventions (EAPCI) and European Association of Acute Cardiac Care (ACCA) endorsed by the Heart Rhythm Society (HRS) and Asia-Pacific Heart Rhythm Society (APHRS). Eur Heart J. 2014; 35: 3155-3179. 